\title{
Effect of a Muscle Relaxant, Chlorphenesin Carbamate, on the Spinal Neurons of Rats
}

\author{
Michio KURACHI and Hironaka AIHARA \\ Research Center, Taisho Pharmaceutical Co.. Ltd., \\ Yoshino-cho 1-403. Ohmiya, Saitama 330, Japan \\ Accepted May 19, 1984
}

\begin{abstract}
The effects of chlorphenesin carbamate (CPC) and mephenesin on spinal neurons were investigated in spinal rats. CPC $(50 \mathrm{mg} / \mathrm{kg}$ i.v. $)$ inhibited the mono(MSR) and poly-synaptic reflex (PSR), the latter being more susceptible than the former to CPC depression. Mephenesin also inhibited MSR and PSR, though the effects were short in duration. CPC had no effect on the dorsal root potential evoked by the stimulation of the dorsal root, while mephenesin reduced the dorsal root-dorsal root reflex. The excitability of motoneuron was reduced by the administration of CPC or mephenesin. The excitability of primary afferent terminal was unchanged by CPC, while it was inhibited by mephenesin. Neither CPC nor mephenesin influenced the field potential evoked by the dorsal root stimulation. Both CPC and mephenesin had no effect on the synaptic recovery. These results suggest that both CPC and mephenesin inhibit the firing of motoneurons by stabilizing the neuronal membrane, while mephenesin additionally suppresses the dorsal root reflex and the excitability of the primary afferent terminal. These inhibitory actions of CPC on spinal activities may contribute, at least partly, to its muscle relaxing action.
\end{abstract}

Mephenesin is a representative centrally acting muscle relaxant. It is, however, rarely employed clinically because of its short duration of action and adverse reactions. Chlorphenesin carbamate (CPC), a drug chemically related to mephenesin (Fig. 1). has been employed clinically because it has a longer lasting effect than mephenesin. Matthews et al. (1) reported that CPC is a selective blocker of polysynaptic pathways at the spinal and supra-spinal levels. In a previous study (2), we found that CPC has an apparent depressant action on the spinal neuron, and it hyperpolarized both the ventral

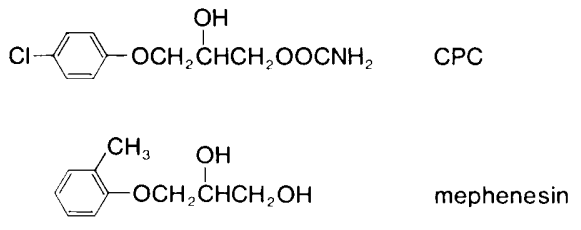

Fig. 1. Chemical structures of chlorphenesin carbamate and mephenesin. and dorsal roots of the isolated frog spinal cord. The purpose of the present study is to investigate the mechanism of action of CPC in the spinal rats, and additionally, to compare the mechanism of CPC with that of mephenesin.

\section{Materials and Methods}

Surgical preparation: Male Wister rats weighing 200-300 g were used. Under ether anesthesia, a tracheal cannula was inserted, and the spinal cord was transected at the $\mathrm{C} 1$ level. Anesthesia was then terminated, and positive pressure ventilation with room air was begun. Animals were treated with minimal amounts of gallamine triethiodide. and supplementary doses were administered as needed. A dorsal laminectomy was performed in the lumbo-sacral region, and both the ventral and dorsal roots below L4 were cut distally at their points of exit from the vertebral column. All exposed surgical areas were covered with liquid paraffin 
maintained at $37 \pm 0.3^{\circ} \mathrm{C}$ by radiant heat. Rectal temperature was maintained at $37 \pm 0.3^{\circ} \mathrm{C}$ using a d.c. heating pad. At least $2 \mathrm{hr}$ was allowed for the preparation to stabilize before electrophysiological experimentation. Since it has been reported that CPC and mephenesin dose-dependently and equipotently affected the spinal function in rats (3) and the administration of $50 \mathrm{mg} / \mathrm{kg}$ i.v. of these drugs resulted in the marked reduction of muscle tone in conscious rats (our preliminary experiment, unpublished observations), the drugs were dissolved in $50 \%$ polyethylene glycol 400 and injected at a dose of $50 \mathrm{mg} / \mathrm{kg}$ through a cannula inserted into the femoral vein in all experiments.

Ventral root potentials: Bipolar platinum electrodes were used for stimulation and recording. A dorsal root (DR) $L 5$ was stimulated with rectangular pulses with $0.05 \mathrm{msec}$ duration and a frequency of $0.2 \mathrm{~Hz}$ (Nihon Kohden, SEN-3201) and with supramaximal potentials which were approx. twice the intensity required to cause maximal reflex response. Mono- and poly-synaptic reflexes (hereafter referred to as MSR and PSR, respectively) were recorded from the ventral root (VR) L5 and displayed on an oscilloscope (Nihon Kohden, VC-9). Five consecutive responses were averaged by an averaging computer (Nihon Kohden, ATAC-350). The MSR and PSR were expressed as the amplitude and the area surrounded by the base line and response, respectively.

Dorsal root potentials: The DRL5 was placed on bipolar platinum electrodes for stimulation (stimulating frequency, $0.2 \mathrm{~Hz}$; pulse duration, 0.05 msec; supramaximal). The dorsal root potentials (DR-DRP) with dorsal root reflex (DR-DRR) were recorded from the DRL4 with a pair of non-polarizable silver-silver chloride electrodes, one of which was placed on the DRL4 close to, but not in contact with, the spinal cord, and the other was placed on the peripheral cut end of the root (intra-electrode distance=approx. 15 $\mathrm{mm}$ ). The dorsal root potentials were amplified by a d.c. amplifier. The amplitudes of five consecutive DR-DRPs with DR-DRR were averaged, and the output was recorded by an inkwriting oscillograph (Nihon Kohden.
WI-130).

Excitability test: The excitability of the primary afferent terminal and motoneuron was measured by Wall's technique (4). A tungsten microelectrode was inserted into the motoneuron pool, which was stimulated by a submaximum current pulse $(0.2 \mathrm{~Hz}$, $0.05 \mathrm{msec}$ ). The antidromic and orthodromic action potentials were recorded with bipolar platinum electrodes on the distal end of the $D R$ and VR, respectively, and five such responses were averaged using the averaging computer.

Field potential: The method used for stimulation and recording was similar to that described by Ono et al. (5). Submaximal rectangular pulses (0.05 $\mathrm{msec}$ duration) were applied to the dorsal rootlet $L 5$ at a frequency of $0.2 \mathrm{~Hz}$. Evoked field potentials were recorded monopolarly with a glass electrode filled with $1 \mathrm{M} \mathrm{NaCl}$, which was lowered into the spinal cord using a micromanipulater. The focal potentials were amplified using a microelectrode amplifier (Nihon Kohden, MEZ-7101), and five such potentials were averaged using the averaging computer.

Synaptic recovery: To assess the time course of functional recovery in the monosynaptic pathway, a method similar to that of Esplin (6) was used. A pair of identical stimuli was applied every $5 \mathrm{sec}$ to the dorsal root. The delay between stimuli in each pair was varied between 5 and $400 \mathrm{msec}$. The amplitudes of the second responses of five consecutive pairs were averaged and expressed as a percentage of the first response.

Statistics: Data were expressed as the mean and standard error of the mean (S.E.M.). Statistical evaluations of data were performed using the two-tailed Student's $t$-test.

Drugs: The drugs used were chlorphenesin carbamate (CPC, Taisho Pharm.), mephenesin (Sigma), gallamine triethiodide (Sigma) and polyethylene glycol 400 (Wako).

\section{Results}

Effects on ventral root responses: Chlorphenesin carbamate (CPC) and mephenesin were intravenously administered at a dose of $50 \mathrm{mg} / \mathrm{kg}$ in all experiments. CPC significantly inhibited PSR, with the maximum 

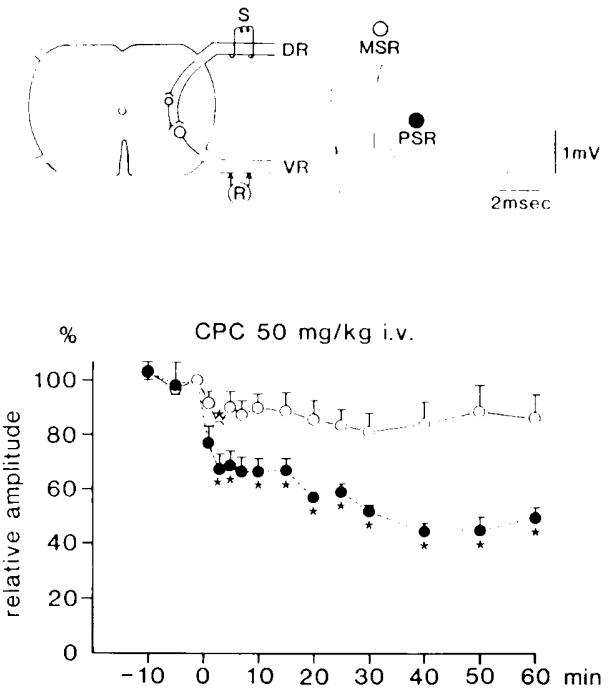
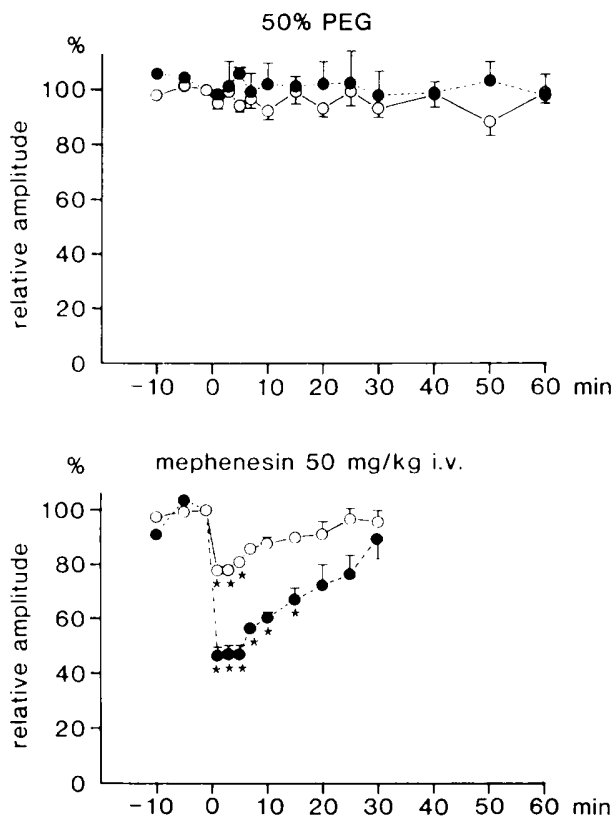

Fig. 2. Effects of chlorphenesin carbamate $(n=7)$ and mephenesin $(n=6)$ on ventral root responses in spinalized (at $\mathrm{C} 1$ level) rats. The schematic inset shows the arrangement of the stimulating and recording electrodes. Stimuli were applied to dorsal root (DR) L5. Reflex responses were recorded from the ipsilateral ventral root (VR) L5. The initial sharply defined component is monosynaptic responses (MSR). It was followed by irregular polysynaptic responses (PSR). Ordinate: the height of MSR (C) and the area of PSR (O) expressed as \% of the value 2 min before the drug or vehicle (50\% PEG) administration. Abscissa: time in min after i.v. injection of the drug or vehicle. Standard errors are indicated by vertical lines. $\star P<0.01$, compared to the vehicle $(n=4)$.

inhibition at 40 min after the drug administration (Fig. 2). Although MSR was also slightly inhibited by CPC, this reduction was not statistically significant except for the value at $3 \mathrm{~min}$ after the administration (Fig. $2)$. On the other hand, mephenesin significantly diminished both MSR and PSR, followed by a recovery to control levels within 30 min after the drug administration (Fig. 2). PSR was found to be somewhat more susceptible than MSR to mephenesin.

Effects on dorsal root potentials: As shown in Fig. 3, CPC administration caused a small reduction in the height of the DR-DRR. The response, however, almost returned to control levels $7 \mathrm{~min}$ after the drug treatment. DRDRP was not affected by CPC. In contrast to CPC, mephenesin remarkably inhibited the DR-DRR and slightly reduced DR-DRP. This inhibitory effect of mephenesin on the DR-DRR completely disappeared $20 \mathrm{~min}$ after the drug administration (Fig. 3).

Effects on the excitability: The influences of CPC and mephenesin on the neuronal excitability are shown in Fig. 4. CPC had no effect on the excitability of the primary afferent terminal, but reduced the excitability of the motoneuron. CPC also strongly depressed the monosynaptic response recorded from the VRL5 (MS, in Fig. 4). The time course of the depression was similar to that observed in MSR elicited by stimulation of the DR (Fig. 2). Mephenesin, unlike CPC, reduced the excitability of the primary afferent terminal (Fig. 4). This drug also depressed the excitability of the motoneuron and the monosynaptic response (Fig. 4).

Effects on field potential: As shown in Fig. 5, the field potential evoked by stimulation of dorsal rootlet $L 5$ consisted of two components, the early one (negative initial potential: NIP) reflecting the presynaptic 

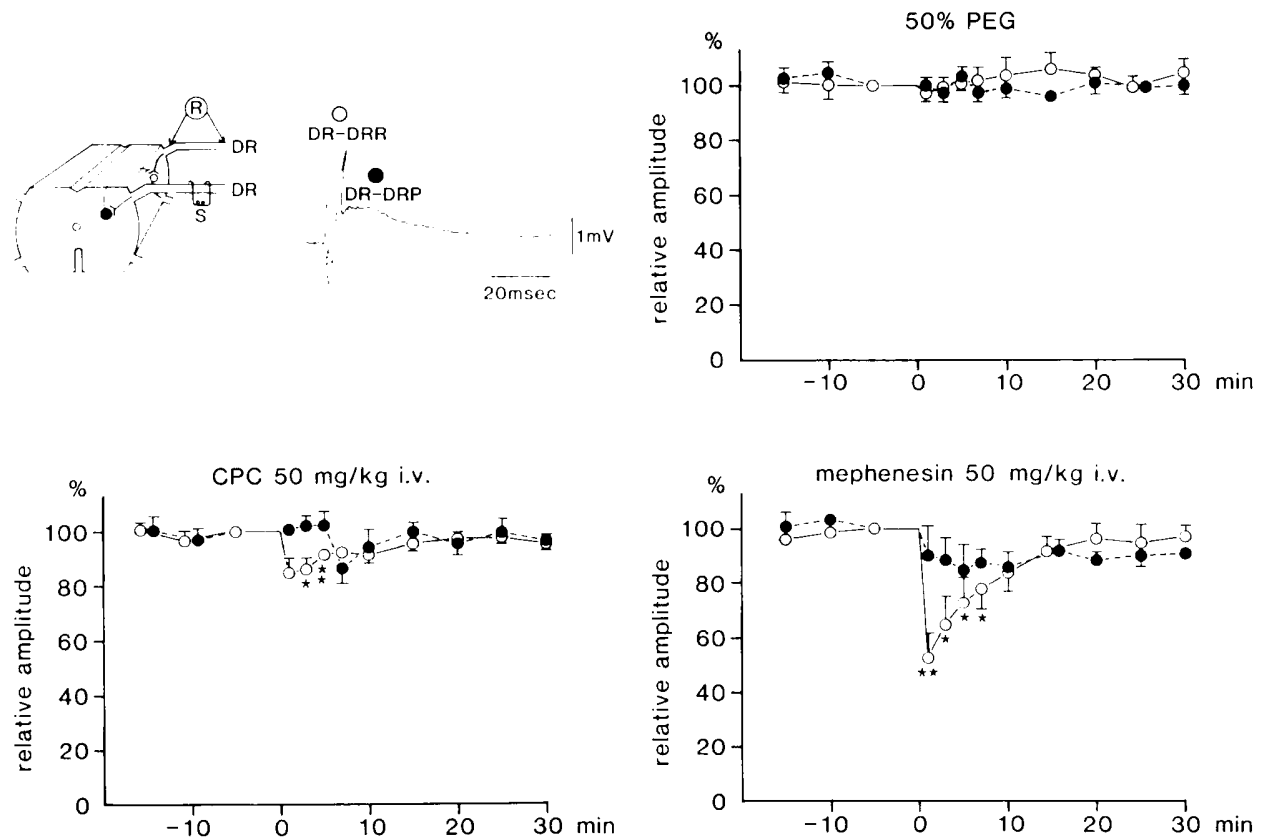

Fig. 3. Effects of chlorphenesin carbamate $(n=5)$ and mephenesin $(n=4)$ on dorsal root potentials. Placement of stimulating and recording electrodes is indicated by the schematic inset: $S$, stimulus: R. recording. The dorsal root-dorsal root reflex (DR-DRR) reflects the firing of the primary afferent terminal: the dorsal root-dorsal root potential (DR-DRP) reflects the primary afferent depolarization. Responses were expressed as \% of the amplitudes of DR-DRR $(O)$ and DR-DRP (O) obtained 5 min before the drug or vehicle $(50 \% \mathrm{PEG})$ administration. Standard errors are indicated by the vertical lines. $\star P<0.05$ or $\star \star P<0.01$, with respect to the vehicle $(n=4)$.

impulses and the other (focal synaptic potential: FSP) reflecting the excitatory postsynaptic potentials (5). Neither CPC nor mephenesin affected the intraspinal field potential during the observed period.

Effects on synaptic recovery: Figure 6 shows the result obtained by applying a conditioning stimulus and an identical test stimulus (paired stimuli technique). At any of the stimulus intervals, there was no difference in synaptic recovery between control and drug treated animals.

\section{Discussion}

The principal findings of the present study are that CPC at a dose of $50 \mathrm{mg} / \mathrm{kg}$ i.v. depressed MSR and PSR (Fig. 2), the DR-DRR (Fig. 3) and the excitability of the motoneuron (Fig. 4); and mephenesin at the same dose depressed MSR and PSR (Fig. 2), the DR-DRR and DR-DRP (Fig. 3), and the excitability of the primary afferent terminal and the motoneuron, these results on mephenesin being coincident with those reported by Ono et al. (5). Accordingly, the mode of action of CPC was different from that of mephenesin in several points, namely. the depression of the DR-DRR and the excitability of the primary afferent terminal. The effect of CPC on the spinal reflex was far longer in duration than that of mephenesin. This can probably be interpreted by the enterohepatic circulation of CPC since the duration of action of CPC for the spinal reflex was shortened in rats with a ligated common bile duct (7).

The intraspinal field potential induced by dorsal root stimulation consists of two components, the negative initial potential (NIP) and the focal synaptic potential (FSP) which reflect the presynaptic impulses propagating in the nerve terminals and the excitatory postsynaptic potentials (EPSP). respectively (5). These components of the 
50\% PEG
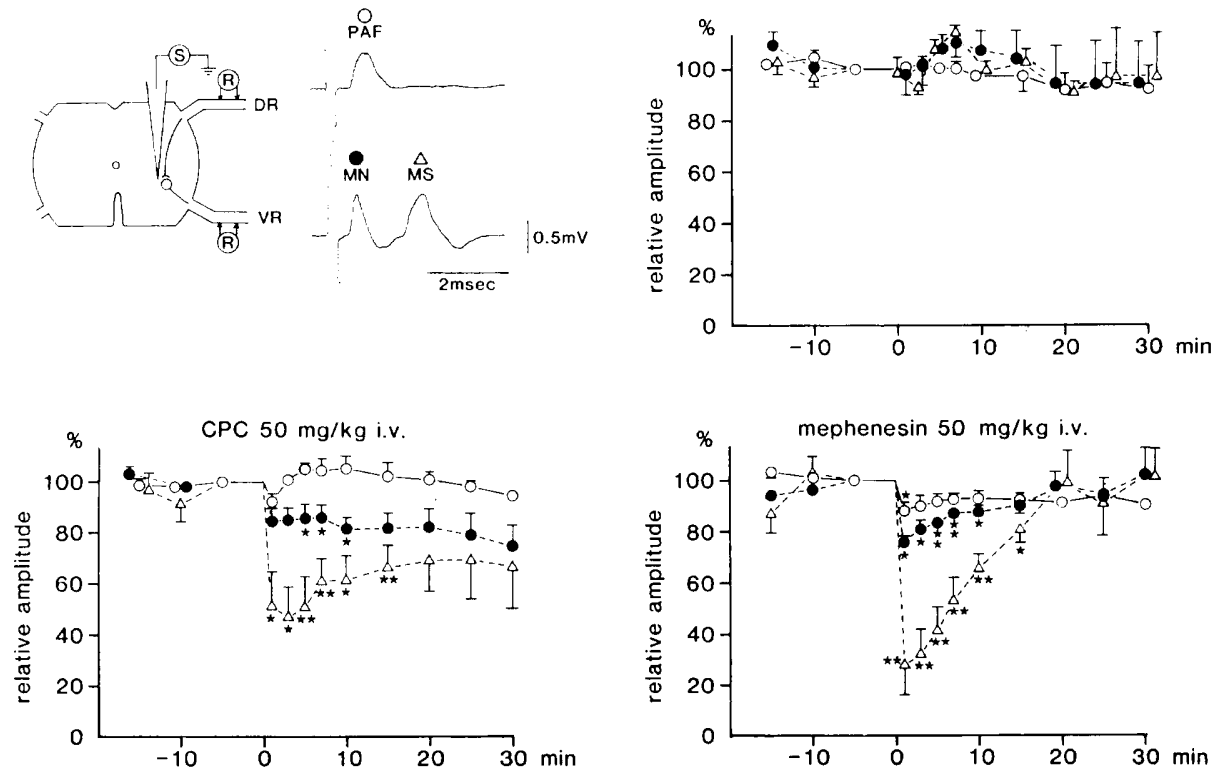

Fig. 4. Effects of chlorphenesin carbamate $(n=5)$ and mephenesin $(n=5)$ on the excitability of primary afferent fiber $(P A F)$ and motoneuron $(M N)$. The arrangement of the stimulating and recording electrodes is shown in the schematic inset. Stimuli were delivered through a tungsten microelectrode inserted into the motoneuron pool. Antidromic and orthodromic responses were recorded from dorsal root (DR) L5. PAF (O), and ventral root (VR) L5. MN $(\mathcal{O})$, respectively. MS $(\triangle)$ indicates the monosynaptically induced response in the motoneuron following interneuron firing. Ordinate: relative amplitude (\%) of the PAF, MN and MS, the value obtained $5 \mathrm{~min}$ before the drug or vehicle (50\% PEG) being taken as $100 \%$. Abscissa: time in min after the drug or vehicle injection. Standard errors are indicated by the vertical lines. $\star P<0.05$ or $\star \star P<0.01$, compared to the vehicle $(n=4)$.
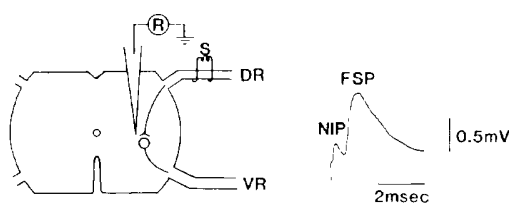

CPC $50 \mathrm{mg} / \mathrm{kg}$ i.v.

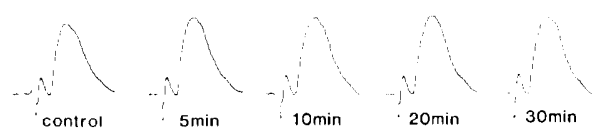

mephenesin 50mg/kg i.v.

$$
\frac{2 \mathrm{msec}}{0.5 \mathrm{mv}}
$$

Fig. 5. Typical examples of focal potentials evoked by submaximal stimulation of the dorsal rootlet in chlorphenesin carbamate- and mephenesin-treated animals. The schematic inset shows the arrangement of stimulating and recording electrodes. The focal potentials were recorded before and 5, 10, 20 and $30 \mathrm{~min}$ after the drug administration. NIP: negative initial potential, FSP: focal synaptic potential.

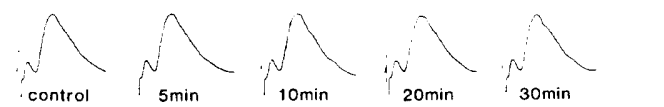



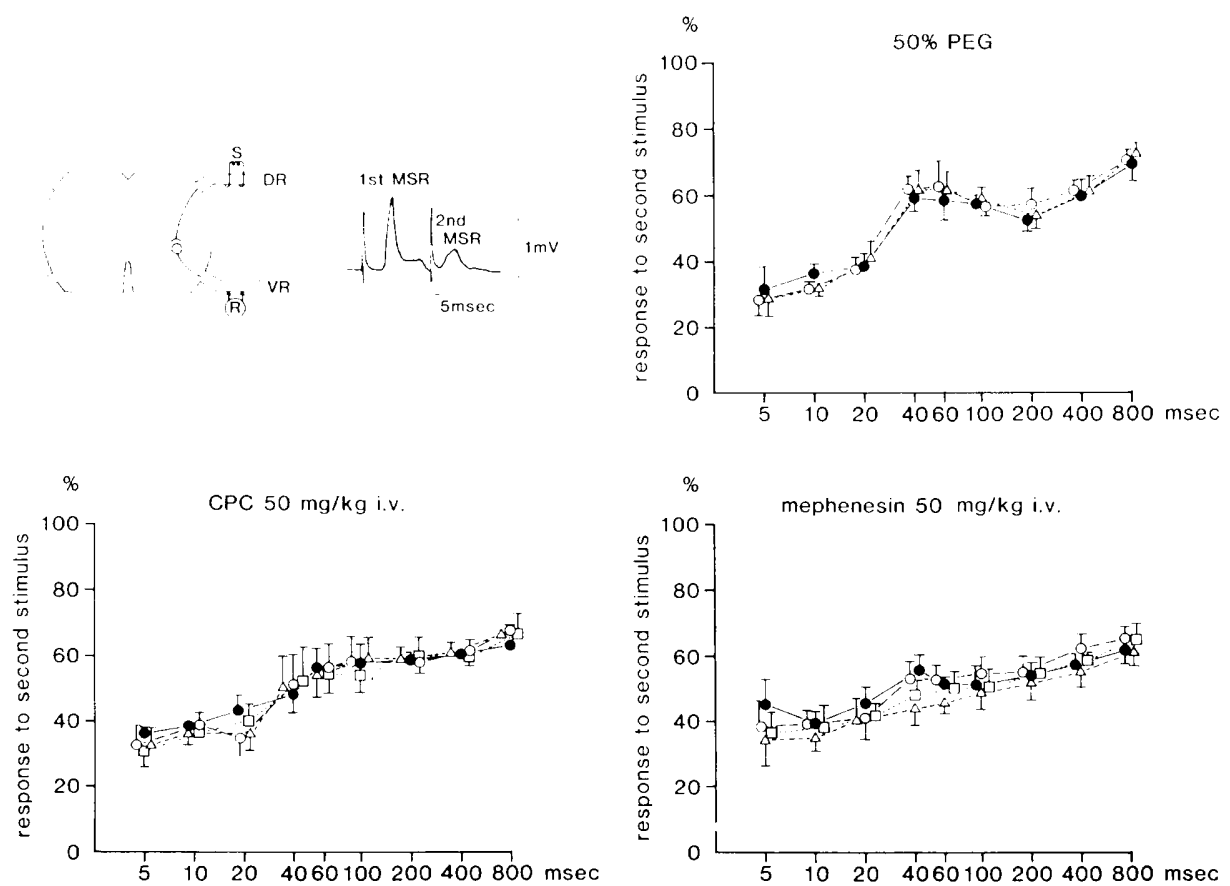

Fig. 6. Effects of chlorphenesin carbamate $(n=6)$ and mephenesin $(n=5)$ on synaptic recovery. Stimulus pairs were applied to the dorsal root (DR) L5 at intervals varied between 5 and 800 msec. The amplitude of the second monosynaptic response ( 2 nd MSR) was plotted as a percentage of the first monosynaptic response (1st MSR) vs. the interval between the paired stimuli. Standard errors are indicated by vertica! lines. Records were performed before $(O)$ and $5(\bigcirc), 20(\triangle)$ and $35 \mathrm{~min}(\square)$ after the drug or vehicle $(50 \%$ PEG, $n=4)$ administration.

field potential were unaffected by CPC and mephenesin (Fig. 5). It is therefore unlikely that these drugs obstruct the invasion of signals into the primary afferent terminal, modulate the release of transmitter(s) and influence the generation of EPSP. The local anesthetic action of CPC and mephenesin at the site of the recording electrode can also be excluded at the dose levels used in this experiment because they did not block the impulse conduction in the primary afferent fiber. It is conceivable, therefore, that the observed depression of MSR and PSR by the drugs (Fig. 2) may result from interference with the mechanism underlying motoneuron firing. The excitability of the motoneuron was found to be lowered by CPC and mephenesin (Fig. 4). It is well-known that the spike potential of a motoneuron is composed of the spikes evoked in the initial segment (IS) and in the soma-dendrites (SD), and the generation of an IS-spike results in the generation of action potentials propagating toward the periphery (8). Accordingly, our results may indicate that $\mathrm{CPC}$ and mephenesin inhibit the motoneuron firing by preventing the generation of the IS-spike or stabilizing the membrane of IS. Since MSR reflects the response of the motoneuron to synchronous afferent impulses, it is inferrable that the blockade of synchronous afferent impulses might stop the firing of the motoneurons.

It is unlikely for CPC to influence presynaptic inhibition since the DR-DRP, which is known to reflect the intensity and time course of presynaptic inhibition (9), was unaffected by this drug. DR-DRR reflects the numbers of spikes generated in the primary afferent terminal. The small reduction of DR-DRR after CPC (Fig. 3) may be attributed to the transient effect on the excitability of the primary afferent terminal (Fig. 4). Mephenesin inhibited both DR-DRP and DR- 
DRR, indicating that the presynaptic inhibition may be affected by this drug.

Both CPC and mephenesin were found not to change the synaptic recovery. Our result was different from that of Esplin (6) who reported the prolongation of synaptic recovery by mephenesin in the cat. Possibly, the synaptic recovery in the rat and cat might be affected differently by mephenesin.

The present study indicates that both CPC and mephenesin inhibit the motoneuron firing by stabilizing the motoneuron. There. however, are several different effects between CPC and mephenesin, namely, mephenesin additionally influenced the presynaptic inhibition and the excitability of the primary afferent terminal. The reduction of motoneuron excitability may explain the clinical effectiveness of CPC in human pathological conditions manifested by the excessive tonic activity of skeletal muscles.

\section{References}

1 Matthews, R.J., Davanso, J.P., Collins, R.J. and Vander Brook, M.J.: The pharmacology of chlorphenesin carbamate. a centrally active muscle relaxant. Arch. Int. Pharmacodyn. Ther. 143, 574-594 (1963)

2 Aihara, H., Kurachi, M., Nakane, S., Sasajima, M. and Ohzeki, M.: The action of chlorphenesin carbamate on the frog spinal cord. Japan. J. Pharmacol. 30, 29-36 (1980)

3 Fukuda, H., Kudo, Y., Ono, $H$. and Kokubo, M.: Pharmacological study on a centrally acting muscle relaxant (chlorphenesin carbamate) with special reference to the effects on motor systems. Folia Pharmacol. Japon. 70, 341-358 (1974) (Abs. in English)

4 Wall, P.D.: Excitability changes in afferent fiber terminations and their relation to slow potentials. J. Physiol. (Lond.) 142, 1-21 (1958)

5 Ono, H., Fukuda, $\mathrm{H}$. and Kudo, Y.: Mechanisms of depressant action of baclofen on the spinal reflex in the rat. Neuropharmacology 18, $647-$ 653 (1979)

6 Esplin. D.W.: Criteria for assessing effects of depressant drugs on spinal cord synaptic transmission with examples of drug selectivity. Arch. Int. Pharmacodyn. Ther. 143, 479-496 (1963)

7 Nozu, T., Suwa, T., Setoyama, K., Kurachi, M. and Tanaka 1.: Influence of the enterohepatic circulation on the metabolism of chlorphenesin carbamate (CPC). 1. Comparison between guinea pig and bile duct-ligated rats. Yakugaku Zassi 97, 1189-1194 (1977) (Abs. in English)

8 Coombs, J.S., Curtis, D.R. and Eccles, J.C.: The generation of impulses in motoneurones. J. Physiol. (Lond.) 139, 232-249 (1957)

9 Eccles, J.C.: The Physiology of Synapses. Academic Press. New York (1964) 